\title{
Hypertension and its determinant factors in a Brazilian male working population Hypertension in male workers
}

Tamyres Araújo Andrade Donato ( $\nabla$ tamyres_donato@hotmail.com )

Universidade Federal da Bahia https://orcid.org/0000-0003-0930-1101

Vanessa Moraes Bezerra

Universidade Federal da Bahia Instituto Multidisciplinar em Saude

Roberta Mendes Abreu Silva

Universidade Federal da Bahia Instituto Multidisciplinar em Saude

Márcio Galvão Guimarães de Oliveira

Universidade Federal da Bahia Instituto Multidisciplinar em Saude

Amanda Cristina de Souza Andrade

Universidade Federal de Mato Grosso

Clavdia Nicolaevna Kochergin

Universidade Federal da Bahia Instituto Multidisciplinar em Saude

Daniela Arruda Soares

Universidade Federal da Bahia Instituto Multidisciplinar em Saude

Danielle Souto de Medeiros

Universidade Federal da Bahia Instituto Multidisciplinar em Saude

José Andrade Louzado

Universidade Federal da Bahia Instituto Multidisciplinar em Saude

Kelle Oliveira Silva

Universidade Federal da Bahia Instituto Multidisciplinar em Saude

Matheus Lopes Cortes

Universidade Federal da Bahia Instituto Multidisciplinar em Saude

Sóstenes Mistro

Universidade Federal da Bahia Instituto Multidisciplinar em Saude

Welma Wildes Cunha Coelho Amorim

Universidade Estadual do Sudoeste da Bahia

Research article

Keywords: hypertension, worker, men, epidemiological studies 
Posted Date: April 27th, 2020

DOI: https://doi.org/10.21203/rs.2.16610/v2

License: (c) (i) This work is licensed under a Creative Commons Attribution 4.0 International License. Read Full License 
The authors have withdrawn this preprint from Research Square 